\title{
The capabilities and scope-of-practice requirements of advanced life support practitioners undertaking critical care transfers: A Delphi study
}

\author{
M Venter, BTEMC; W Stassen, MPhil EM \\ Department of Emergency Medical Care, Faculty of Health Sciences, University of Johannesburg, South Africa
}

Corresponding author: W Stassen (stassen88@gmail.com)

\begin{abstract}
Background. Critical care transfers (CCT) refer to the high level of care given during transport (via ambulance, helicopter or fixed-wing aircraft) of patients who are of high acuity. In South Africa (SA), advanced life support (ALS) paramedics undertake CCTs. The scope of ALS in SA has no extended protocol regarding procedures or medications in terms of dealing with these CCTs.

Aim. The aim of this study was to obtain the opinions of several experts in fields pertaining to critical care and transport and to gain consensus on the skills and scope-of-practice requirements of paramedics undertaking CCTs in the SA setting.

Methods. A modified Delphi study consisting of three rounds was undertaken using an online survey platform. A heterogeneous sample $(n=7)$, consisting of specialists in the fields of anaesthesiology, emergency medicine, internal medicine, critical care, critical care transport and paediatrics, was asked to indicate whether, in their opinion, selected procedures and medications were needed within the scope of practice of paramedics undertaking CCTs.

Results. After three rounds, consensus was obtained in 70\% (57/81) of procedures and medications. Many of these items are not currently within the scope of paramedics' training. The panel felt that paramedics undertaking these transfers should have additional postgraduate training that is specific to critical care.

Conclusion. Major discrepancies exist between the current scope of paramedic practice and the suggested required scope of practice for CCTs. An extended scope of practice and additional training should be considered for these practitioners.

S Afr J Crit Care 2016;32(2):58-61. DOI:10.7196/SAJCC.2016.v32i2.275
\end{abstract}

There is currently a critical shortage of intensive and specialist care in South Africa (SA). ${ }^{[1]}$ In fact, $77 \%$ of state healthcare facilities and $16 \%$ of private healthcare facilities do not have intensive or high-care capacity. ${ }^{[1]}$ Owing to this shortage, adequate district and provincial healthcare might not be available in many areas, ${ }^{[2]}$ necessitating frequent transfer of patients to urban tertiary centres for specialist care.

Internationally, paramedics undertaking critical care transfers (CCTs) have extended scope of practice when compared with standard emergency paramedics in order to be equipped for these high-acuity patients. ${ }^{[3]}$ Locally, however, all paramedics registered as advanced life support (ALS) practitioners may undertake CCTs. ${ }^{[4]}$ No extended training or additional scope of practice is given to these paramedics who might need to intervene to stabilise patients for transfer ${ }^{[4]}$ Studies have shown that adverse events (AEs) during CCTs are significant and proportionate to the level of training and experience of the transferring practitioner. ${ }^{[5,6]}$

A prospective 1-year audit conducted in SA in 2001 focused on CCTs of paediatric patients in a low-resource setting. ${ }^{[7]}$ The study found that the transfers were predominantly undertaken by paramedics (82\%) via road ambulance $(76 \%){ }^{[7]}$ It was found that a number of technical AEs such as inadequate monitoring, absent venous access, and malpositioning of endotracheal tubes occurred in 36\% of all the review cases. ${ }^{[7]}$ Clinical AEs such as shock, hypoxia or hypoglycaemia occurred in $27 \%$ of the cases. ${ }^{[7]}$ In addition, critical AEs including cardiac arrest were noted in $9 \%$ of the cases. ${ }^{[7]}$ Ultimately, it was concluded that these AEs could be significantly reduced by further training of ALS paramedics and use of specialised teams for paediatric CCTs. ${ }^{[7]}$

Although the current scope of practice of paramedics in SA is extensive, it does not account for the critical care patient specifically. The aim of this study was to investigate the opinions of several experts in fields pertaining to critical care and transport and to obtain consensus on the skills and scope of practice requirements of paramedics undertaking CCTs in the SA setting.

\section{Methods}

A modified Delphi study consisting of three rounds was undertaken using the online survey platform Google Forms (Google Inc, USA). A purposive and heterogeneous sample $(N=12)$ consisting of specialists in the fields of anaesthesiology, emergency medicine, internal medicine, critical care, critical care transport (intensive care unit (ICU)-transfer paramedics), and paediatrics was used. An a priori consensus level was set at $75 \% .{ }^{[8]}$ Paediatric and adult skill sets were separated and required to be evaluated independently because of the AE and difficulty profiles of procedures in these two populations. The proposed procedures and medications were chosen using an existing international protocol $^{[3]}$ for critical care paramedics and contextualising these to the local burden of disease and resource constraints. Respondents were asked to justify their views in free text fields, and these responses were subjected to content analysis. Anonymous feedback was then included in the subsequent rounds.

\section{Results}

Twelve potential participants were approached, of whom seven agreed to participate. An attrition rate of $14.3 \%$ was seen between round one and round two, leaving six 
responses eligible for analysis at the end of round three. The final panel therefore consisted of two ICU transfer paramedics, one paediatrician, one intensivist, one anaesthesiologist and one emergency physician. All participants were asked to respond to and comment on both the paediatric and adult procedures included in the study.

A total of 81 procedures and medications were proposed to the panel for review, of which 14 were newly proposed by the panel during the first round and reviewed during the second and third rounds. Consensus of $65.7 \%$ (44/67 proposed procedures and medications) was obtained during the first round. The total level of consensus gained was $70.3 \%$ $(n=57)$ after three rounds. A total of 24 procedures and medications did not gain consensus after the third round. The study was terminated after three rounds because of time and resource constraints.

Tables 1 and 2 show all paediatric and adult procedures proposed to the panel for consideration, respectively, while Table 3 presents all the proposed medications. The shaded items indicate the procedures and medications that gained consensus.

\section{Content analysis}

For each of the skills, the panel had the option to motivate their views. The panel felt that because of a relative scarcity of healthcare providers in SA, CCTs are inevitable in our setting. This was related back to the need for paramedics transporting critical care patients to have a

\section{Table 1. Paediatric procedures}

Arterial blood gas analysis

Arterial line insertion

Arterial line monitoring

Blood analysis of infection markers

Blood analysis of renal function

Cardiac 12-lead ECG

Chest X-ray interpretation

CT and MRI interpretation

Initiation of blood product transfusion

Initiation of tracheostomy

Intracranial pressure monitoring

Management of high-frequency oscillatory ventilation*

Mechanical ventilation $<5 \mathrm{~kg}$

Monitoring of chest drainage system

Monitoring of tracheostomy

Multichannel infusion pump/syringe

Non-invasive ventilation for neonates (i.e. nasal CPAP)*

Placement of chest drainage system

Point-of-care ultrasound

Pulmonary artery catheter removal

RSI

Transport isolette - care of a neonate

Vascular access - arterial stab

Vascular access - central venous catheter

${ }^{*}$ Procedures suggested by members of the panel. Shaded areas indicate procedures that gained positive consensus.

ECG = electrocardiograph; $\mathrm{CT}=$ computed tomography; $\mathrm{MRI}=$ magnetic

resonance imaging; $\mathrm{CPAP}=$ continuous positive airway pressure; $\mathrm{RSI}=$ rapid sequence intubation.
Table 2. Adult procedures

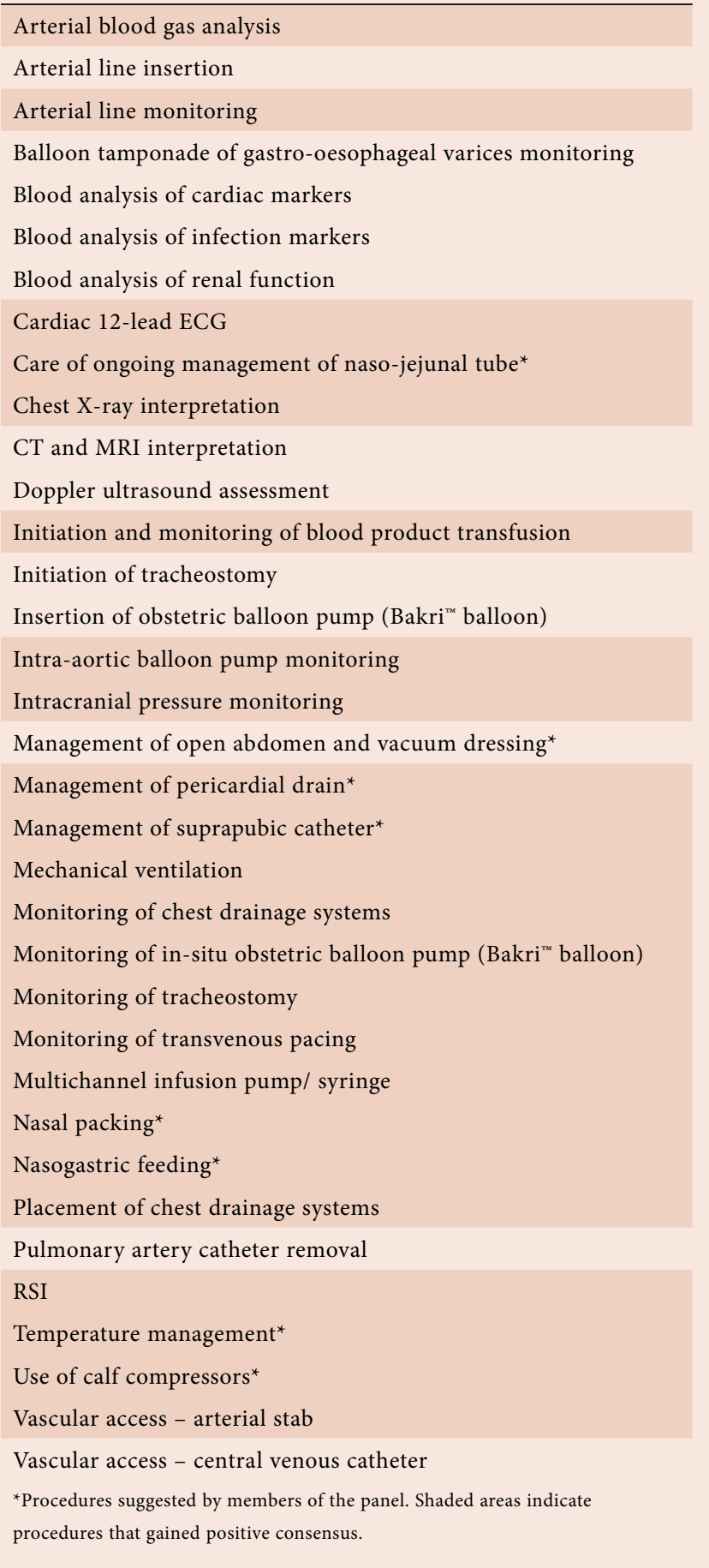

broadened scope of practice because of referring hospitals not having physicians available at all times to perform some necessary procedures or administer certain necessary medications to the critically ill patient prior to transport. The panel agreed that these additional skill sets and scope should be guided by clinical policy and be subjected to clinical governance and peer review.

The panel was asked who they felt should be undertaking CCT in SA. The panel felt that the emergency care practitioner (ECP, degree paramedics) with postgraduate experience and ICU training would be most appropriate: 'At least an ECP ... with some form of critical care specific postgraduate training...' Furthermore the panel added that 'multidisciplinary teams... doctors, nurses and paramedics...' may bring 


\section{Table 3. Medication}

All vaccinations*
Anaesthesia/induction agent (propofol)
Analgesia (paracetamol, fentanyl, remifentanil,
dexmedetomidine)
Antibiotics
Anticoagulants
Anti-emetics
Antihyperglycaemic agents
Antihypertensives
Antimalarials
Antiprotozoals
Antipsychotics
Artificial surfactant
Beta-blockers
Diuretics (mannitol)
Inotropes/vasopressors (dopamine, dobutamine, and
phenylephrine)
Misoprostol ${ }^{*}$
Neuromuscular blocking agents (paralytics)
Oxytocin ${ }^{*}$
Post-exposure antiretrovirals ${ }^{*}$
Prostin
Proton-pump inhibitors
Thrombolytics
${ }^{*}$ Medications suggested by members of the panel. Shaded areas indicate
medications that gained positive consensus.

added benefit to certain patients during transport.

Numerous panellists stated that an additional course should be created for paramedics in order to gain additional knowledge and skills for the CCT environment. It was stated that doctors, nurses, or paramedics should not undertake these (critical care) transfers without additional and regular refresher training.' It was added that it would be 'imperative ... to broaden the scope of the ECP paramedic, which is completely to the patient's benefit'.

\section{Discussion}

The panel felt that paramedics who undertake CCTs of high-acuity patients should have an extended scope of practice and additional training to utilise this scope safely. Most notably, this extended scope pertained to additional diagnostic capability, insertion and monitoring of indwelling devices, and further extension of the paramedic's ability to administer a wider range of anaesthetic, analgesic and cardiac medications, among others. Further specialist training for paramedics was unanimously suggested by the panel.

Owing to low resources, especially in the public sector, many patients do not have access to specialist healthcare services based on their geographic locale or their socioeconomic status. ${ }^{[1,9,10]}$ International and local data have shown that outcomes improve when patients are referred to regional specialised centres where a multidisciplinary team may manage their ailment. ${ }^{[1-13]}$ For this reason, many patients will require CCT. In SA, paramedics currently do not have additional training to undertake these specialised transfers. ${ }^{[4]}$
Locally, there are three streams and different qualifications in order to become a paramedic. ${ }^{[14]}$ Firstly, after completing a basic and intermediate course and gaining some practical experience, a 9 12-month course (Critical Care Assistant Certificate) may be completed, allowing for registration with the Health Professions Council of South Africa (HPCSA) as a paramedic. Alternatively, paramedic registration may be obtained by completing a 3-year National Diploma course. Finally, a 4-year honours degree can be undertaken, allowing for the individual to register as an ECP (as opposed to paramedic) with the HPCSA. ${ }^{[14]}$ Regardless of the stream chosen to obtain registration to practise, there is considerable variation between qualifications and even different educational institutions (offering the same qualification) in the exposure and training in critical care transport. This ranges from a 10-week module in critical care (including lectures and practical ICU ward rotations) to absolutely no teaching beyond the most basic elements of mechanical ventilation and the use of medication infusion devices. However, despite this variability in training and critical care exposure, all paramedics are expected to transfer critical care patients ${ }^{[4]}$ - often for protracted periods. ${ }^{[1]}$

Studies have shown that AEs during CCTs are significant and proportionate to the level of training and experience of the transferring practitioner. ${ }^{[5,6]}$ A literature review in 2015, based on 33 recently published studies on complications during CCTs of adult patients, found that inter-hospital transfers had an AE rate as high as $34 \% .^{[5]}$ This appears to be corroborated by a local study among paediatric admissions at a tertiary hospital which showed an AE rate of between $27 \%$ and $36 \%{ }^{[7]}$ Further to this, a local paper published as early as 2001 a recommendation that transferring practitioners receive additional training in the care of neonates to avoid in-transit AE. ${ }^{[15]} \mathrm{AE}$ rates seem to be related to the stability of the patient before transfer. ${ }^{[1,16]}$ Critical care transport teams often spend a considerable amount of time stabilising the patient for transfer - their main purpose being to ensure patient stability in order to avoid in-transit events. ${ }^{[16]}$ Unless the patient has a time-critical pathology (e.g. ST segment elevation myocardial infarction (STEMI), acute trauma requiring surgery, stroke), taking the time to optimise the patient's haemodynamic and respiratory status prior to transport has been found to improve morbidity and mortality. ${ }^{[16,17]}$ In fact, one study showed a shorter hospital stay when paramedics stayed longer at the referring facility stabilising the patient. ${ }^{[18]}$

In order to achieve patient stability, a variety of procedures and medications must be administered by the CCT team. ${ }^{[16-18]}$ The current paramedic scope of practice locally is mainly focused on emergency, rather than critical care. ${ }^{[4]}$ Often paramedics are left to initiate or monitor medication infusions that are outside of their scope of practice (e.g. dobutamine in cardiogenic shock, or prostaglandins in ductal-dependant lesions). These interventions may be lifesaving, yet paramedics are not trained to use them, nor licensed to carry them. The expert panel in this study identified and recommended a myriad of additional skills and medications that they felt would be necessary during critical care transport.

In contrast, internationally, paramedics have the opportunity to further specialise in critical care transport by completing postgraduate courses. ${ }^{[19]}$ These courses provide a better clinical and theoretical understanding of the critical care patient and environment, as well as the appropriate management of these patients in comparison with pre-hospital treatment. ${ }^{[19]}$ Critical care paramedics have an extended scope of practice regarding procedures, such as initiation of blood product transfusions, rapid sequence intubation, and up to 60 additional medications in comparison with the average paramedic. ${ }^{[3]}$ 


\section{Limitations and recommendations}

Despite having fair heterogeneity in the representation of different specialties within this study, the sample size was small. In addition, an attrition of $14.3 \%$ further decreased the sample size. It is suggested that a similar study be conducted on a larger scale to be more representative of the different specialties involved. We also recommend consultation with key role-players to inform the development of a curriculum for critical care transport and formalisation of further education to this end. Regulatory bodies, such as the HPCSA, should also consider expanding the current registers to include a specialty registration after completion of the specialisation course. Further specialty training should also be considered for those undertaking the CCT of neonatal and paediatric patients in order to minimise in-transport AEs.

\section{Conclusion}

The results of the study suggest that paramedics who are currently undertaking CCTs locally, often over long distances, are not equipped with the necessary skills and scope of practice to overcome AEs during these transfers.

Owing to resource constraints, it is unlikely that local CCTs will be completed by physicians in the near future. SA paramedics and ECPs currently have variable and insufficient training and exposure to critical care to safely complete these patient transfers. This has been shown to increase AEs, and morbidity and mortality. It is still unclear what additional training these practitioners will require to become specialists in this field; however, it is clear that further training is necessary. Training should not only be based on international practices, but should also take into consideration the unique SA burden of disease and the health referral system of the country.

\footnotetext{
References

1. Scribante J, Bhagwanjee S. National audit of critical care resources in South Africa: Transfer of critically ill patients. S Afr Med J 2007;97(12):1323-1326.
}

2. Kautzky K, Tollman SM. A perspective on primary health care in South Africa: In: South African Health Review 2008. Durban: Health Systems Trust, 2008:17-30.

3. Paramedic Association of Canada. National Occupational Competency Profile for Paramedics. Ontario: Paramedic Association of Canada, 2011 (updated October 2011). http://www.paramedic. ca/uploaded/web/documents/2011-10-31-Approved-NOCP-English-Master.pdf (accessed 12 April 2016).

4. Professional Board for Emergency Care. Capabilities of Emergency Care Providers. Pretoria: Health Professions Council of South Africa, 2013 (updated July 2013). http://www.hpcsa.co.za/ Uploads/editor/UserFiles/downloads/emergency_care/capabilities_baa_eae_ecp_ect_jul_2013_ Uploads/editor/UserFiles/downloads/
final.pdf (accessed 13 April 2016).

5. Bambi S, Lucchini A, Innocenti D, Mattiussi E. Complications in critically ill adult patients transportations reported in the recent literature. Emerg Care J 2015;11(1):12-18. http://dx.doi. org/10.4081/ecj.2015.4781

6. Fan E, MacDonald RD, Adhikari NKJ, et al. Outcomes of interfacility critical care adult patien transport: A systematic review. Crit Care 2006;10(1):1-7. http://dx.doi.org/10.1007/bf03022513

7. Hatherill M, Waggie Z, Reynolds L, Argent A. Transport of critically ill children in a resourcelimited setting. Intensive Care Med 2003;29(9):1547-1554. http://dx.doi.org/10.1007/s00134003-1888-7

8. Powell C. The Delphi technique: Myths and realities - methodological issues in nursing research. J Adv Nurs 2003;41(4):376-382. http://dx.doi.org/10.1046/j.1365-2648.2003.02537.x

9. Harris B, Goudge J, Ataguba JE, et al. Inequities in access to health care in South Africa. J Public Health Policy 2011;32:S102-123. http://dx.doi.org/10.1057/jphp.2011.35

10. Snyders A, Delport R. Referral pathways for reperfusion of STEMI - developing strategies for appropriate intervention. SA Heart 2015;12(2):74-80.

11. Ali SF, Singhal AB, Viswanathan A, Rost NS, Schwamm LH. Characteristics and outcomes among patients transferred to a regional comprehensive stroke center for tertiary care. Stroke 2013;44(11):3148-3153. http://dx.doi.org/10.1161/strokeaha.113.002493

12. Michaels AJ, Hill JG, Long WB, et al. Adult refractory hypoxemic acute respiratory distress center. Am J Surg 2013;205(5):492-498. http://dx.doi.org/10.1016/j.amjsurg.2013.01.025

13. Schoon MG. Impact of inter-facility transport on maternal mortality in the Free State Province. S Afr Med J 2013;103(8):534-537. http://dx.doi.org/10.7196/samj.6828

14. MacFarlane C, van Loggerenberg C, Kloeck W. International EMS systems in South Africa - past, present, and future. Resuscitation 2005;64(2):145-148. http://dx.doi.org/10.1016/j. resuscitation.2004.11.003

15. Hadley GP, Mars M. Improving neonatal transport in the Third World - technology or teaching? S Afr J Surg 2001;39(4):122-124.

16. Wilcox SR, Saia MS, Waden H, et al. On-scene times for inter-facility transport of patients with hypoxemic respiratory failure. Prehosp Disaster Med 2016;31(3):1-5. http://dx.doi.org/10.1017/ s1049023x16000315

17. Gebremicheal M, Borg U, Habasi NM, et al. Interhospital transport of the extremely ill patient: The mobile intensive care unit. Crit Care Med 2000;28:79-85. http://dx.doi.org/10.1097/00003246200001000-00013

18. Belway D, Dodek PM, Keenan SP, Norena M, Wong H. The role of transport intervals in outcomes for critically ill patients who are transferred to referral centers. J Crit Care 2008;23(3):287-294 http://dx.doi.org/10.1016/j.jcrc.2007.04.007

19. University of Florida. Critical Care Paramedic Certificate (updated 1 March 2016). http://www. bcn.ufl.edu/academics/certificates/ccp/ (accessed 12 April 2016). 